\title{
Characterization of sequence elements from Malvastrum yellow vein betasatellite regulating promoter activity and DNA replication
}

Jie Zhang, Xinyue Zhang, Yaqin Wang, Huwei Hou and Yajuan Qian*

\begin{abstract}
Background: Many monopartite begomoviruses are associated with betasatellites, but only several promoters from which were isolated and studied. In this study, the $\beta C 1$ promoter from Malvastrum yellow vein betasatellite (MYVB) was characterized and important sequence elements were identified to modulate promoter activity and replication of MYVB.

Results: A 991 nucleotide (nt) fragment upstream of the translation start site of the $B C 1$ open reading frame of MYVB and a series of deletions within this fragment were constructed and fused to the $\beta$-glucuronidase (GUS) and green fluorescent protein (GFP) reporter genes, respectively. Agrobacterium-mediated transient expression assays showed that the $991 \mathrm{nt}$ fragment was functional and that a $28 \mathrm{nt}$ region (between -390 nt and -418 nt), which includes a $5^{\prime}$ UTR Py-rich stretch motif, was important for promoter activity. Replication assays using Nicotiana benthamiana leaf discs and whole plants showed that deletion of the $5^{\prime} U T R$ Py-rich stretch impaired viral satellite replication in the presence of the helper virus. Transgenic assays demonstrated that the $991 \mathrm{nt}$ fragment conferred a constitutive expression pattern in transgenic tobacco plants and that a $214 \mathrm{nt}$ fragment at the $3^{\prime}$-end of this sequence was sufficient to drive this expression pattern.
\end{abstract}

Conclusion: Our results showed that the $\beta C 1$ promoter of MYVB displayed a constitutive expression pattern and a 5'UTR Py-rich stretch motif regulated both $\beta C 1$ promoter activity and MYVB replication.

Keywords: Begomovirus, Betasatellite, Malvastrum yellow vein virus, Promoter

\section{Background}

The Geminiviridae are a family of plant DNA viruses whose members are classified into four genera: Mastrevirus, Begomovirus, Curtovirus and Topocuvirus. The majority of geminiviruses belong to the genus Begomovirus. Begomoviruses are either monopartite or bipartite in the organization of their genome and many monopartite begomoviruses are associated with betasatellites (formerly called DNA $\beta$ ) [1-3]. Betasatellites depend on the helper begomoviruses for replication, encapsidation and insect transmission as well as spread within and between plants [2,4]. Comparison of the nucleotide sequences from all known betasatellite molecules reveals three conserved features: a highly conserved region

\footnotetext{
* Correspondence: yjaian@zju.edu.cn

State Key Laboratory of Rice Biology, Institute of Biotechnology, Zhejiang University, Hangzhou 310029, People's Republic of China
}

called the satellite conserved region (SCR), a single gene (known as $\beta C 1$ ) that is conserved in both position and size and is a determinant of symptoms, and an A-rich region [5-7].

Using Agrobacterium-mediated transient expression and stable transformation system, many motifs/ sequences have been identified to be involved in regulation of geminivirus transcription [8-10]. For example, Shung et al. [11] identified two elements located upstream of AL1935 and AL1629, important for transcription of complementary sense RNAs derived from Tomato golden mosaic virus (TGMV). A region located between $-125 \mathrm{nt}$ and $-60 \mathrm{nt}$ from the transcription start site in the TGMV $C P$ promoter was reported to be involved in both activation and derepression by TrAP $[12,13]$. However, few promoters from betasatellites have been isolated and studied since Guan and Zhou [14] first reported the characterization of the $\beta C 1$ promoter of the

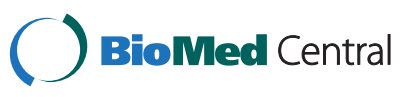


Tomato yellow leaf curl China betasatellite (TYLCCNB) and subsequently Eini et al. [10] identified sequence elements which regulated $\beta C 1$ transcription associated with the Cotton leaf curl Multan betasatellite (CLCuMB). Malvastrum yellow vein virus (MYVV) is a typical monopartite geminivirus. Previous reports have shown that the betasatellite associated with MYVV (MYVB) is involved in symptom induction and it is required for enhancing the accumulation of helper virus in tobacco plants [15]. In order to further elucidate the transcriptional regulation and replication of the MYVB, in this study, we have characterized the putative promoter of the $\beta C 1$ gene of MYVB using both transient and stable transgenic expression approaches. Furthermore, we have identified a motif consisting of a $5^{\prime} \mathrm{UTR}$ Py-rich stretch important for MYVB replication.

\section{Results}

Analysis of the putative promoter sequence of the MYVB $\beta C 1$ gene

The sequence of the putative promoter encompassing the entire non-coding region (991 nt) upstream of the MYVB $\beta C 1$ open reading frame was analyzed using the PlantCARE program (http://bioinformatics.psb.ugent.be/ webtools/plantcare/html/). As illustrated in Figure 1, a number of putative regulatory motifs and cis-elements were predicted, including a typical TATA-box ( $-37 \mathrm{nt})$, some CAAT-boxes (-58 nt, $-106 \mathrm{nt},-984 \mathrm{nt})$ and a Gbox $(-144 \mathrm{nt})$. Strikingly, compared with previously reported $\beta C 1$ promoters $[10,14]$, the MYVB $\beta C 1$ promoter displayed key differences in the composition of the putative promoter. Of particular interest, was a 5'UTR Py-rich stretch, which usually plays an important role in increasing gene expression [16-18].

\section{Identification of cis-elements regulating $\beta C 1$ expression}

To determine the cis-elements responsible for the transcriptional control of MYVB $\beta C 1$, the $991 \mathrm{nt}$ fragment and a series of deletions within that sequence were constructed and fused to a promoter-less pINT121 vector (Figure 2). As a positive control, the pINT121 vector containing the GUS gene driven by the Cauliflower mosaic virus (CaMV) 35S promoter was used. Following Agrobacterium-mediated transient expression in tobacco leaves, fluorometric assays suggested that the promoter deletion constructs exhibited varying GUS expression levels (Figure 3A). The $991 \mathrm{nt}$ fragment $(\mathrm{p} \beta \mathrm{C} 1)$ drove the greatest GUS expression that was approximately $29 \%$ of that observed in tissues infiltrated with the CaMV $35 \mathrm{~S}$ promoter. Deletion of the region from -991 to -419

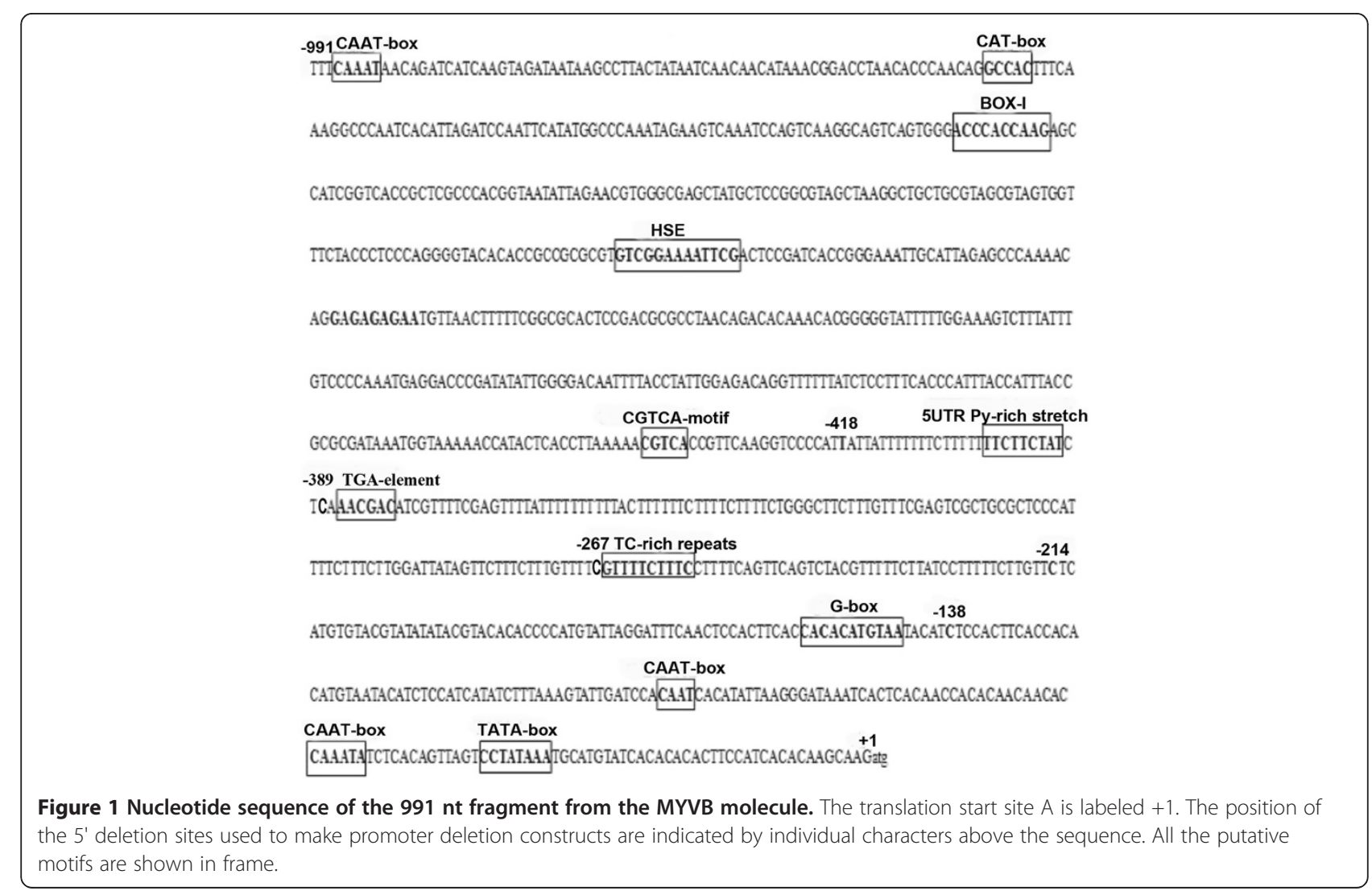




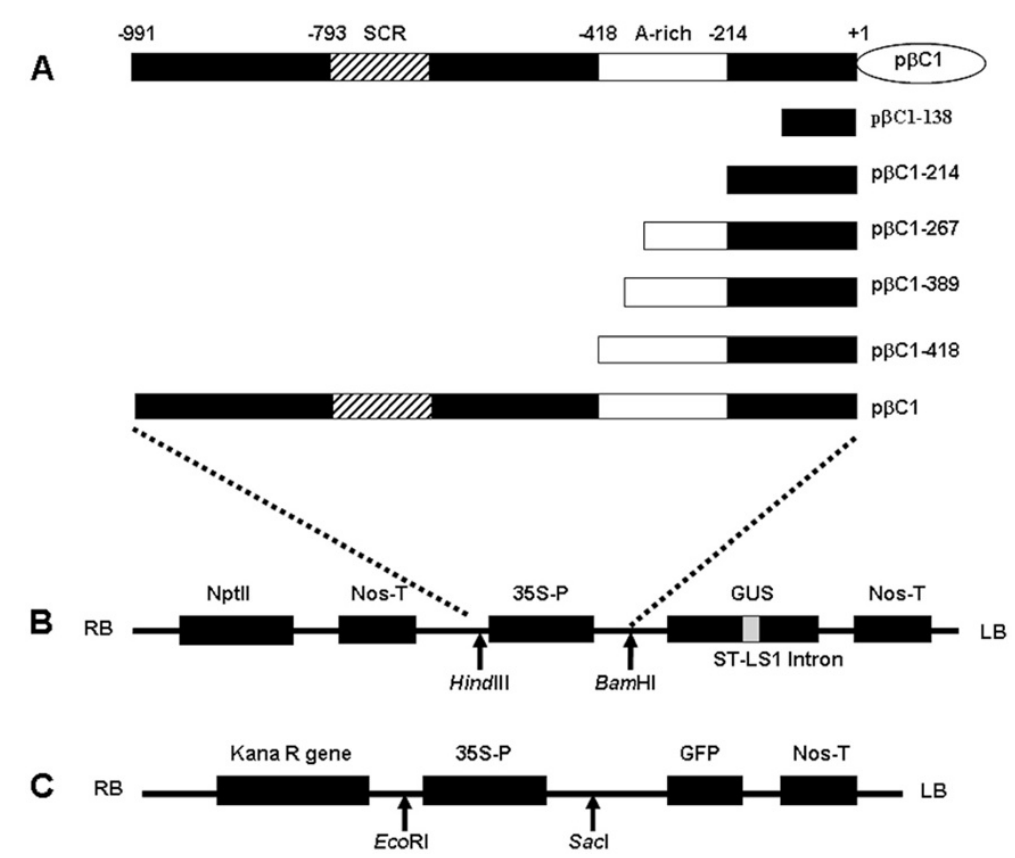

Figure 2 Construction of different $\beta C 1$ promoters of MYVB. The satellite conserved region (SCR) and A-rich region are shown in boxes. The translation start site is numbered +1 . (A) Schematic representation of the MYVB genome and various $\beta C 1$ promoters. (B) The promoters were cloned into the pINT121 binary vector. (C) The promoter constructs were cloned into the pCHF3:GFP vector.

nt in the $\mathrm{p} \beta \mathrm{C} 1-418$ construct resulted in only a minor difference in GUS expression levels compared with $\mathrm{p} \beta C 1(P>0.05)$. Interestingly, deletion of the region from -991 to $-390 \mathrm{nt}(\mathrm{p} \beta C 1-389)$ resulted in a marked reduction in GUS expression levels to just $5 \%$ of that observed for the CaMV $35 \mathrm{~S}$ promoter, while there were no significant differences among $\mathrm{p} \beta C 1-389, \mathrm{p} \beta C 1-267$ and $\mathrm{p} \beta C 1-214(P>0.05)$. It is worth noting that deletion from -991 to $-139 \mathrm{nt}(\mathrm{p} \beta \mathrm{C} 1-138)$ led to almost complete loss of GUS activity (Figure 3A).

In order to further identify the cis-elements involved in the transcriptional control of $\beta C 1$, different promoter deletion sequences were inserted individually upstream of the GFP reporter gene within the expression vector pCHF3:GFP. The results revealed that $64 \mathrm{~h}$ after infiltration, significant differences in the intensity of GFP fluorescence were observed among the various constructs. As illustrated in Figure 3B, compared with other constructs, $\mathrm{p} \beta C 1$ and $\mathrm{p} \beta C 1-418$ produced relatively high levels of fluorescence, but much lower levels compared with the positive control pCHF3:GFP. GFP fluorescence was also observed to be produced from constructs $\mathrm{p} \beta C 1-389$ to $\mathrm{p} \beta C 1-214$, while the fluorescence of $\mathrm{p} \beta \mathrm{C} 1-138$ was almost identical to that of the negative control pCHF3. Calculation of the fluorescence intensity revealed that the sequence within a $214 \mathrm{nt}$ region upstream of the translation start site was fundamentally required for $\beta C 1$ promoter activity (Figure 3A). These results were consistent with those of the fluorometric GUS assay.

\section{A 5'UTR Py-rich stretch motif regulates $\beta C 1$ promoter activity}

Figure 3 showed that deletion of the region from -991 to $-390 \mathrm{nt}$ in the $\mathrm{p} \beta \mathrm{C} 1-389$ resulted in a remarkable reduction in promoter activity compared with $\mathrm{p} \beta \mathrm{C} 1-418$, which indicated the presence of a positive cis-element in the region between -390 to $-418 \mathrm{nt}$ of the MYVB $\beta C 1$ promoter. Further sequence alignment analysis revealed the presence of a $5^{\prime} \mathrm{UTR}$ Py-rich stretch in this region. Therefore, the entire non-coding region promoter construct excluding the 5'UTR Py-rich stretch motif $(p \beta C 1 \Delta U T R)$ was obtained. Sixty-four hours after infiltration into leaves of $N$. benthamiana plants, fluorometric assays revealed that relative GUS activity of the $\mathrm{p} \beta C 1 \Delta \mathrm{UTR}$ declined to $11 \%$ of that driven by the CaMV $35 \mathrm{~S}$ promoter, which differed significantly from that of $\mathrm{p} \beta \mathrm{C} 1(P<0.01)$.

\section{Roles of the 5'UTR Py-rich stretch motif in MYVB replication and pathogenicity}

To investigate the involvement of this $5^{\prime} \mathrm{UTR}$ Py-rich stretch motif in MYVB replication, the full-length MYVB sequence excluding the $5^{\prime} U T R$ Py-rich stretch motif (MYVBAUTR) and a tandem direct repeat of MYVBAUTR were produced in the binary vector $\mathrm{pBIN-}$ PLUS. The infectious clone of MYVBAUTR was tested for replication in $N$. benthamiana leaf discs assays using MYVV as the helper virus. Southern blot analysis showed that deletion of the 5'UTR Py-rich stretch motif 


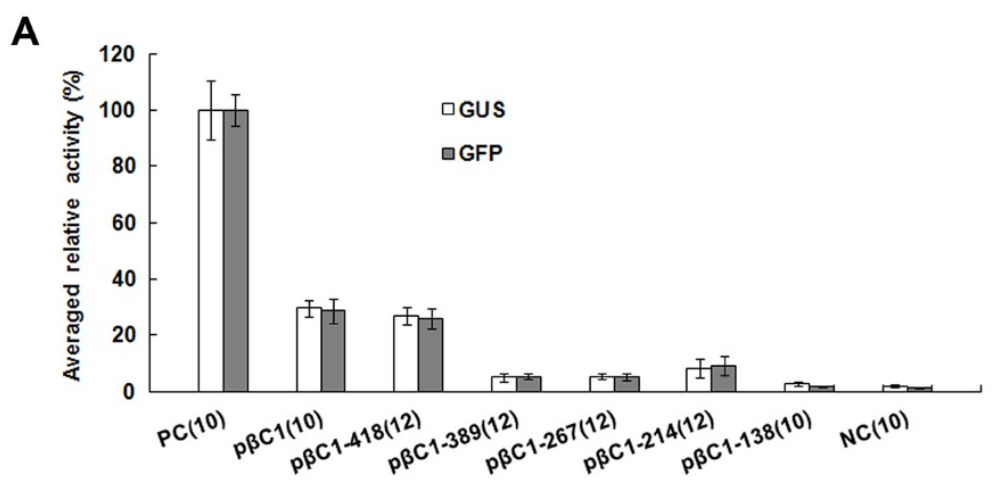

B

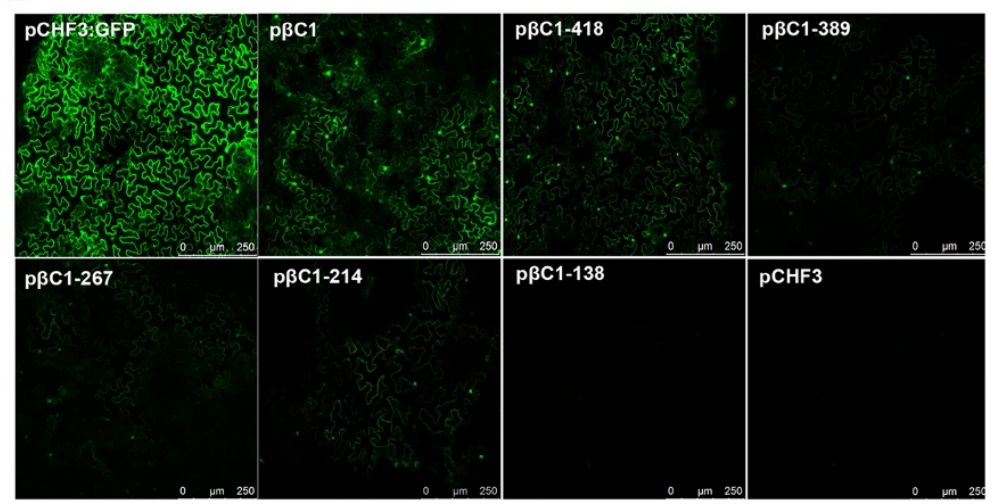

Figure 3 Fluorometric activity analysis in $\mathbf{N}$. benthamiana leaves after transient expression of pINT121 and pCHF3:GFP. (A) The mean GUS or GFP activity from the CaMV 35 S promoter of pINT121 or pCHF3:GFP was arbitrarily assigned as 100\% and used to standardize the activity of all other constructs. The standard deviation of each construct is shown with error bars. The number of replicates of each sample is indicated in parentheses. PC, positive control, binary vector pINT121 or pCHF3:GFP; NC, negative control, promoter-less vector pBINGUS or pCHF3 vector without the GFP gene. (B) Fluorometric GFP activity analysis in N. benthamiana leaves after transient expression driven by various $\beta C 1$ promoter constructs. pCHF3:GFP and pCHF3 were used as positive and negative controls, respectively.

resulted in a significant reduction in replication of both the betasatellite and helper virus (Figure $4 \mathrm{~B}$ and $4 \mathrm{C}$ ). The intensity value obtained from MYVV plus MYVB was arbitrarily assigned as $100 \%$. The average normalized intensity values of blot bands are presented in Figure 4C. The results showed that deletion of the $5^{\prime}$ UTR Py-rich stretch motif decreased viral DNA or betasatellite accumulation to $58 \%$ and $65 \%$, respectively, compared with that of MYVV plus MYVB.

The roles of the $5^{\prime}$ UTR Py-rich stretch motif in viral replication and symptom development were assayed in whole plants. Infectious clones of MYVBAUTR or MYVB together with MYVV were inoculated into $N$. benthamiana plants. Both MYVBAUTR and MYVB caused systemic infection in all plants tested, with similar symptoms including downward leaf curling as well as vein yellowing observed 30 days post-inoculation (dpi) (Figure 5A). The presence of viral DNA was determined by Southern hybridization analysis (Figure $5 \mathrm{~B}$ and $5 \mathrm{C}$ ). The results showed that the accumulation of the deleted MYVB was decreased to $62 \%$ compared with that of wild-type MYVB. Furthermore, a slight decrease in the accumulation of MYVV in tissues co-infected with MYVBAUTR plus MYVV was observed, equivalent to $89 \%$ of that observed in tissues co-infected with MYVB plus MYVV (Figure 5B and 5C).

\section{Evaluation of the expression pattern in tobacco}

In order to determine the spatial expression pattern driven by the putative promoter from the MYVB $\beta C 1$ gene, the $\mathrm{p} \beta C 1$ and $\mathrm{p} \beta C 1-214$ constructs were introduced into tobacco plants via Agrobacterium-mediated transformation. Three independent transgenic lines of each construct with relatively high GUS activity were selected for histochemical analysis. In general, the transgenic $\mathrm{p} \beta C 1$ and $\mathrm{p} \beta C 1-214$ lines conferred the same expression pattern, with blue staining observed in almost all tissues of the roots, stems and leaves (Figure 6). In root sections prepared from $\mathrm{p} \beta C 1$ and $\mathrm{p} \beta C 1-214$ transgenic plants, GUS staining was observed both in the vascular cylinder and the root cap region (Figure 6D and 6J). In stem cross sections, GUS expression driven by 


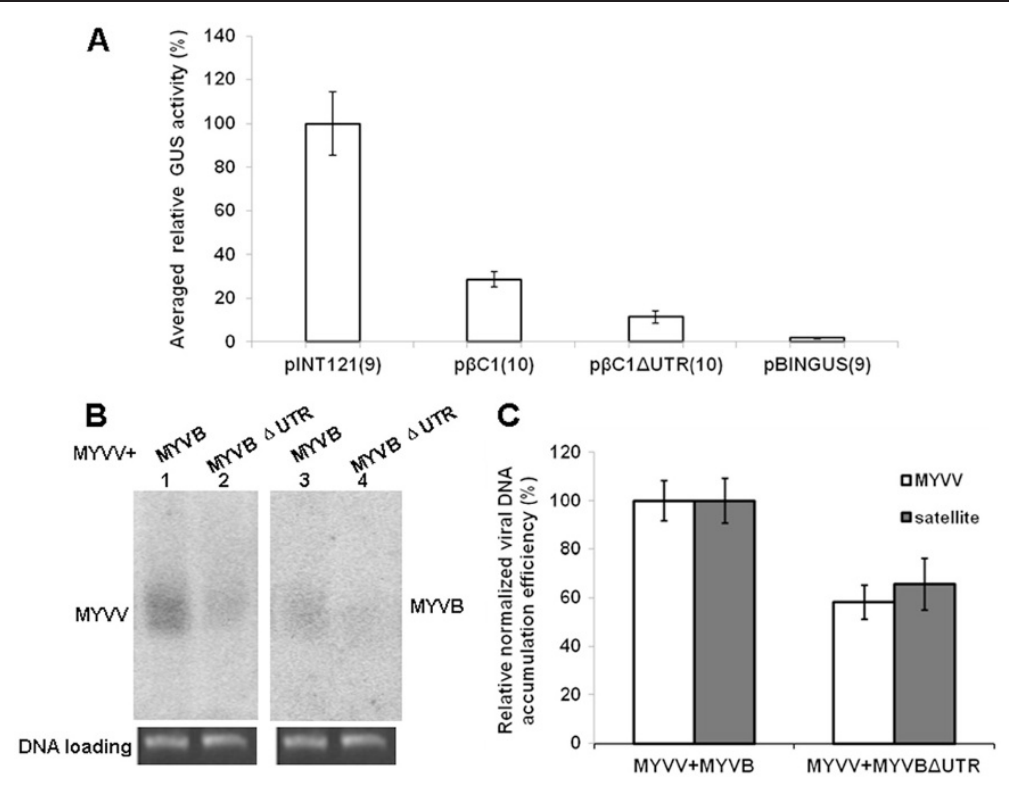

Figure 4 The effect of the $5^{\prime}$ UTR Py-rich stretch motif on MYVB promoter activity and MYVB replication. (A)

Fluorometric GUS activity analysis of the mutant promoter construct in N. benthamiana leaves. pINT121 and pBINGUS were used as positive and negative controls, respectively, and GUS activity of pINT121 was arbitrarily assigned as 100\%. The number of replicates of each sample is indicated in parentheses. (B) Southern blot of total nucleic acid extracts of tobacco leaf co-inoculated with infectious clones of MYW plus MYVB (lanes 1 and 3), or MYV plus MYVBAUTR (lanes 2 and 4). Blots were probed with either the CP gene sequence of MYV (lanes 1 and 2) or the full-length MYVB sequence (lanes 3 and 4). Photographs of the ethidium bromide-stained gels are shown below the blot as an indication of loading control. (C) Densitometric quantification of band intensities. The value obtained from MYW plus MYVB was arbitrarily assigned as 100\%. Standard deviation values are based on three independent experiments.

$\mathrm{p} \beta C 1$ and $\mathrm{p} \beta \mathrm{C} 1-214$ was observed mainly in parts of xylem parenchyma as well as internal and external phloem cells (Figure 6A and 6I). The GUS staining signal in $\mathrm{p} \beta C 1$ and $\mathrm{p} \beta C 1-214$ leaf sections was also found in different cell types, including palisade mesophyll cells, spongy mesophyll cells and the secondary vascular bundle (Figure 6C and 6H).

\section{Discussion}

In this study, a $991 \mathrm{nt}$ fragment upstream of the translation start site of $\beta C 1$ of MYVB was identified as the promoter and the 214 nt fragment from the $3^{\prime}$ end of the 991 nt fragment, which contains a G-box, was shown to be involved in basic promoter activity. Promoter activity was almost abolished in the $138 \mathrm{nt}$ fragment from the $3^{\prime}$ end of the 991 nt without a G-box. These results suggested that the G-box acts as a positive regulatory element in the control of MYVB $\beta C 1$ transcription. Previous reports have indicated that G-box elements present in promoter regions of several geminiviruses and some plant genes bind to host factors involved in activating transcription [14,19]. Recently Eini et al. [10] also identified a $68 \mathrm{nt}$ fragment containing a G-box upstream of the $\beta C 1$ gene associated with $\mathrm{CLCuMB}$ that was found to be important in the regulation of promoter activity. Furthermore, the G-box motif was shown to bind specifically to proteins in nuclear extracts from tobacco leaf tissues. We postulate that MYVB $\beta C 1$ shares a similar transcription regulation mechanism with other organisms although further investigations are required to elucidate the interaction of the MYVB G-box motif with host nuclear factors.

Previous evidence has shown that the $5^{\prime} \mathrm{UTR}$ Py-rich stretch motifs are highly transcription level-related sequence elements regulating the activity of various promoters [16-18]. As shown in Figure 4A, our results also demonstrated that site-directed deletion of the $5^{\prime} \mathrm{UTR}$ Py-rich stretch within the 991 nt $\beta C 1$ promoter sequence resulted in a $60 \%$ reduction in promoter activity compared with the intact $\beta C 1$ promoter, indicating the involvement of this element in the transcriptional regulation of $\beta C 1$. In both fungi and animals, although transcription and DNA replication are divided into different biological processes, they frequently share the same regulatory elements [20-23]. Sequences/motifs involved in transcription or DNA replication have been detected in some geminiviruses [9,10,24-26]. Tu and Sunter [26] identified a conserved binding site within the TGMV AL-1629 promoter, which is necessary for efficient viral DNA replication. Previous studies that showed that betasatellites depend on the helper begomoviruses for replication $[5,15]$. However, up to date, the mechanism 


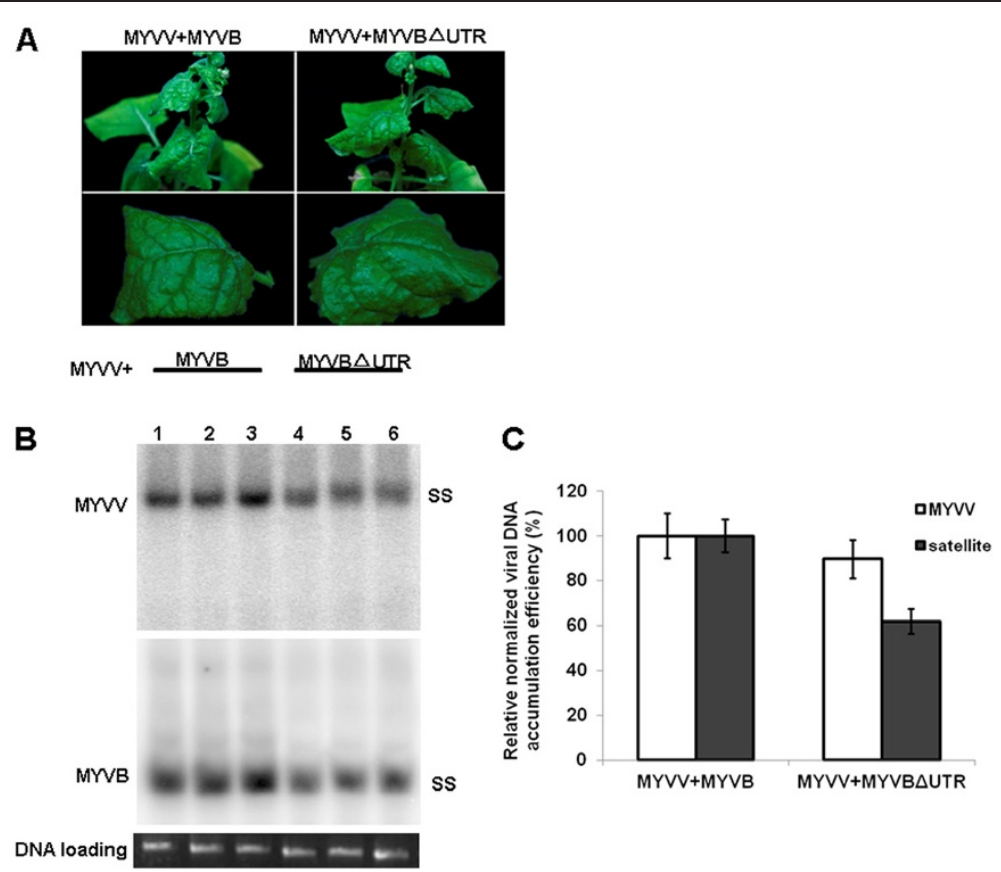

Figure 5 Infectivity and symptoms induced by MYVV together with MYVB or MYVBAUTR. (A) Symptoms induced by infectious clones of MYV plus MYVB or MYVBAUTR on N. benthamiana plants at 30 dpi. (B) Southern blot analysis of viral and betasatellite DNAs in inoculated N. benthamiana plants. Nucleic acids were extracted from upper leaves of plants infected with MYV and MYVB (lanes 1-3), MYW and MYVBDUTR (lanes 4-6). Blots were probed with the CP gene sequence of MYV (top) or full-length of MYVB (bottom). The positions of single-stranded DNA (SS) forms are indicated. The lower panel represents an ethidium bromide-stained gel of DNA samples as a loading control. (C) Densitometric quantification of band intensities. The value obtained from MYV plus MYVB was arbitrarily assigned as 100\%. Standard deviation values are based on three independent experiments.

of interaction of begomovirus-encoded Rep with betasatellites to initiate satellite replication was not fully understood as well as betasatellites lack the iteron sequences encoded by their helper viruses. In this study, infectious assays in leaf discs showed that the 5'UTR Py- rich stretch motif also has an important role in MYVB replication.

Mutagenesis of the TYLCCNB and Tobacco Curly Shoot betasatellite ( $\mathrm{TbCSB}$ ) showed that the $\beta C 1$ protein is the symptom determinant, although the promoter of
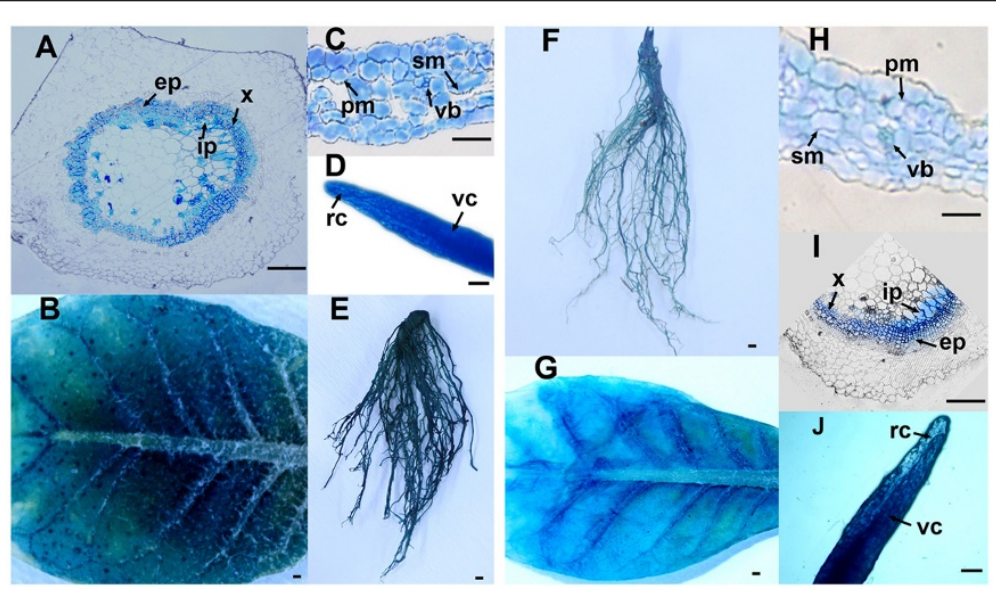

Figure 6 Histochemical localization of GUS expression patterns of $\mathrm{p} \beta C 1$ (A-E) and $\mathrm{p} \beta C 1-214$ (F-J) in transgenic N. tabacum plants. (A, I) Transverse sections of stem, (B, G) underside of the leaf, $(\mathbf{C}, \mathbf{H})$ transverse sections through the leaves, $(\mathbf{D}, \mathbf{J})$ longitudinal sections of the roots, and (E, F) root. Abbreviations: rc. root cap; vc. vascular cylinder; x. xylem; ph. phloem; ep. external phloem; ip. internal phloem; v. vein; pm. palisade mesophyll; sm. spongy mesophyll; vb. vascular bundle. Scale bars, $20 \mu \mathrm{m}$. 
$\beta C 1$ has some influence on symptom induction [27]. In our experiments, despite the production of low levels of betasatellite accumulation, the truncated MYVBAUTR had no marked effect on the viral symptoms compared with the wild-type MYVB. Taken together, it is suspected that $5^{\prime}$ UTR Py-rich stretch motif is involved in regulating the replication of betasatellite but is indispensable for viral symptom development.

Among these characterized geminivirus promoters, some are able to drive constitutive gene expression in transgenic plants, while others have more specific patterns of expression $[8,14,28,29]$. Histochemical staining assays revealed that the $991 \mathrm{nt}$ fragment and the $214 \mathrm{nt}$ fragment containing a G-box conferred a constitutive expression pattern. Previous studies have indicated that a $955 \mathrm{nt}$ fragment upstream of the translation start site of the $\beta C 1$ gene from TYLCCNB is a phloem-specific promoter [14]. Sequence analysis showed that the two putative promoters encompassing the entire non-coding region upstream of the $\beta C 1$ open reading frame of MYVB and TYLCCNB shared only $42 \%$ nucleotide sequence identity. An ASL box and a TATGAAC motif, which are thought to be responsible for the phloemspecific expression [30], were absent in the promoter region of MYVB. It can be speculated that sequence differences result in the different tissue expression patterns driven by betasatellite promoters.

In conclusion, the MYVB $\beta C 1$ promoter directs a constitutive expression pattern in tobacco plants and might be suitable for special plant genetic engineering studies of low-level gene expression.

\section{Methods}

\section{Construction of plant expression vectors}

A series of primers (Table 1) designed according to the MYVB genome (GenBank accession no. AJ421482), were used to amplify the putative promoter region of the $\beta C 1$ gene using the previously constructed MYVB infectious clone as the template [15]. The PCR-amplified fragments were cloned into the pGEM-T easy vector (Promega) for sequencing and then digested individually with HindIII/ BamHI or EcoRI/SacI restriction enzymes. The resulting fragments were inserted into the corresponding sites within the binary vector pINT121 or pCHF3: GFP to replace the original CaMV $35 \mathrm{~S}$ promoter, producing the following expression constructs: $\mathrm{p} \beta \mathrm{C} 1, \mathrm{p} \beta \mathrm{C} 1-418, \mathrm{p} \beta \mathrm{C} 1-$ 389, $\mathrm{p} \beta C 1-267, \mathrm{p} \beta C 1-214$ and $\mathrm{p} \beta C 1-138$ (Figure 1). The plasmid pINT121 or pCHF3:GFP, in which the reporter gene is driven by the $35 \mathrm{~S}$ promoter, was used as a positive control. For the negative control, pBINGUS (consisting of the GUS-Nos fragment excised from the $B a m \mathrm{HI} / E c o$ RI sites of pINT121 and inserted into the $B a m H I / E c o R I$ sites of the pBINPLUS vector) and pCHF3 without the GFP gene were used, respectively.

The 5'UTR Py-rich stretch motif $(\beta C 1 \Delta \mathrm{UTR})$ was deleted from the entire non-coding region of the $\beta C 1$ gene using an overlap-extension PCR strategy [27]. Two independent PCRs were conducted with primer pairs, Y47 $\beta p-F / Y 47 \beta \Delta U T R-R$ and Y47 $\beta \Delta U T R-F / Y 47 \beta p-R$ and PCR products were subsequently added to the standard PCR system and the flanked primer pair Y47 $\beta \mathrm{p}-\mathrm{F} /$ $\mathrm{Y} 47 \beta \mathrm{p}-\mathrm{R}$ was added to amplify the completed $\beta C 1 \Delta$ UTR. The resulting fragments were digested with HindIII/BamHI and inserted into the corresponding sites within the binary vector pINT121 to produce the expression construct, $\mathrm{p} \beta \mathrm{C} 1 \Delta \mathrm{UTR}$. Using the same overlap-extension PCR strategy, the full-length MYVB sequence with deletion of the $5^{\prime} \mathrm{UTR}$ Py-rich stretch (MYVBAUTR) was obtained. An infectious clone containing MYVBAUTR was produced as described in [15]. The complete monomeric sequence of MYVB $\Delta U T R$ was amplified using primers $\beta 01 / \beta 02$. The fragment was then inserted into the pGEM-T easy vector (Promega) to produce the clone, pGEM-MYVBAUTR. Subsequently,

Table 1 Sequences of primers used for the PCRs

\begin{tabular}{|c|c|c|c|}
\hline Primers & Sequences (5'-3') & Underlined restriction site & Position on MYVB \\
\hline Y47ßp138-F & GAATTCAAGCTTCTCCATCATATCTITAAAGT & EcoRl, Hindlll & $690-671$ \\
\hline Y47ßp214-F & GAATTCAAGCTTCTCATGTGTACGTATATATACG & EcoRl, HindllI & $766-745$ \\
\hline Y47ßp267-F & GAATTCAAGCTTCGTTITCTTTCCTITTCAGTTCAG & EcoRl, Hindlll & 819-796 \\
\hline Y47ßp389-F & GAATTCAAGCTTCAAACGACATCGTTTTCGAGTT & EcoRl, Hindlll & $941-920$ \\
\hline Y47ßp418-F & GAATTCAAGCTITTATTATITITTCTIIIITCTTC & EcoRl, Hindlll & $971-947$ \\
\hline Y47ßp-F & GAATTCAAGCTIITCAAATAACAGATCATCAAG & EcoRl, Hindlll & $195-174$ \\
\hline Y47ßp-R & GAGCTCGGATCCCTTGCTTGTGTGATGGAAGTG & $\mathrm{SaCl}, \mathrm{BamHI}$ & $553-573$ \\
\hline$Y 47 \beta \Delta U T R-F$ & TTTTCTTTTTCTCAAACGACATCGTITCG & - & 962-953 plus 943-924 \\
\hline$Y 47 \beta \Delta U T R-R$ & GTCGTTTGAGAAAAAGAAAAAAATAATAATGGGG & - & 934-943 plus 953-976 \\
\hline$\beta 01$ & GTAGGTACCACTACGCTACGCAGCAGCC & Kpnl & 1290-1308 \\
\hline$\beta 02$ & AGTGGTACCTACCCTCCCAGGGGTACAC & Kpnl & $1283-1265$ \\
\hline$\beta 03$ & GTAGAAACCACTACGCTACGCAGCAGCC & - & 1290-1308 \\
\hline
\end{tabular}


another copy of the complete MYVBAUTR sequence was amplified using primers $\beta 03 / \beta 02$ to produce pGEM-MYVB $\triangle U T R$ '. The pGEM-MYVB $\triangle U T R$ clone was digested with $K p n \mathrm{I}$ and inserted into the unique $K p n I$ site of pGEM-MYVB $\triangle \mathrm{UTR}^{\prime}$ to produce pGEM$2 M Y V B \Delta U T R$. Then pGEM-2MYVBAUTR was digested with EcoRI and inserted into the binary vector pBINPLUS to produce pBIN-2MYVBAUTR, which contains a tandem dimeric repeat of MYVBAUTR molecules. Infectious clones of MYVV and MYVB were produced previously [15].

Expression vectors were introduced individually into Agrobacterium tumefaciens strain EHA105 as described previously [3].

\section{Transient expression assay}

Transient expression analysis by Agrobacterium-mediated delivery into plants was carried out as described previously [31]. Three independent experiments were carried out for each construct.

\section{Fluorometric GFP assay}

Leaves of 4 week-old $N$. benthamiana plants were infiltrated with the A. tumefaciens harboring the various expression constructs fused to the GFP marker gene. Approximately $64 \mathrm{~h}$ after infiltration, $1 \mathrm{~cm}^{2}$ leaf fragments were excised and GFP fluorescence was examined in epidermal cells by confocal laser scanning microscopy (CLSM, Leica TCS SP5, Mannheim, Germany).

\section{Analysis of replication in leaf discs and infected plants}

A. tumefaciens strain EHA105 harboring either helper virus or betasatellite infectious clones was used for infection of $N$. benthamiana leaf discs [32] or the whole plants [5] as previously described. Total DNA was extracted using the CTAB method from leaf discs after 6 days or from co-inoculated plants after 30 days. Approximately $10 \mu \mathrm{g}$ of total DNA was blotted and hybridized with ${ }^{32} \mathrm{P}$-dCTP randomly labeled DNA probes specific for MYVV or MYVB [15]. The band intensities were quantified using Image J software [33].

\section{Plant transformation}

Agrobacterium-mediated transformation of Nicotiana tabacum leaf discs was conducted according to a previously published procedure [34]. Transformants were selected on Murashige and Skoog medium containing 100 $\mu \mathrm{g} / \mathrm{ml}$ kanamycin and $500 \mu \mathrm{g} / \mathrm{ml}$ carbenicillin. Regenerated kanamycin-resistant plants were grown on a rooting medium and then transferred to soil after confirmation by PCR using specific primers for GUS gene (5'-ATGT TACGTCCTGTAGAAACC-3'/5'-TCATTGTTTGCCTCC CTGC-3').

\section{Fluorometric GUS assay and histochemical staining of GUS}

$N$. benthamiana leaves were sampled $64 \mathrm{~h}$ after infiltration and ground in Passive Lysis Buffer (Promega) using a pestle and mortar. Supernatants obtained after centrifugation were used for fluorometric assays. Protein content of the samples was determined by an Eppendorf BioPhotometer (Eppendorf, Hamburg, Germany) using BSA as a standard. Quantitative GUS fluorometric assays were conducted essentially as described by Jefferson et al. [35] and using a Perkin-Elmer LS50B luminescence spectrometer (excitation at $365 \mathrm{~nm}$ and emission at $455 \mathrm{~nm}$ ) to measure the fluorescence of 4-methylumbelliferone (4-MU), which is formed as a result of the cleavage of 4-methylumbelliferyl$\beta$-D-glucuronide (MUG). GUS activity was calculated as the production of 4-MU from MUG in picomoles per minute per microgram of protein. The mean GUS activity from the CaMV 35S promoter of pINT121 was arbitrarily assigned as $100 \%$ and used to standardize the activities for all of the other constructs. The resulting data were analyzed using the LSD method of SPSS v12.0 software (SPSS, Chicago, IL, USA).

For the histochemical detection of GUS activity, fresh plant tissue from several transgenic $N$. tabacum was incubated for 3 to $12 \mathrm{~h}$ in a 5-bromo-4-chloro-3-indolyl $\beta$-D-glucuronide staining solution at $37^{\circ} \mathrm{C}$ as described by Jefferson et al. [35]. The stained samples were cleared by several washes with $70 \%$ ethanol and then embedded as described previously [36]. A 11800 Pyramitome (LKBBROMMA, Stockholm, Sweden) was used for slicing tissue into semi-thin sections. Images of stained sections were photographed with an OLYMPUS BH-2 stereomicroscope (OLYMPUS, Japan).

\section{Competing interests}

The authors declare that they have no conflict of interests.

\section{Authors' contributions}

$J Z, X Z, Y W$ and $H H$ performed the experiments. YQ conceived the study. JZ, $X Z$ and $Y Q$ wrote the manuscript. All authors read and approved the final manuscript.

\section{Acknowledgments}

This work was supported by grants from the National transgenic Research Projects of China (No. 2009ZX08009-134B) and the National Natural Science Foundation of China (No. 30770092).

Received: 8 March 2012 Accepted: 1 October 2012

Published: 11 October 2012

\section{References}

1. Briddon RW, Bull SE, Amin I, Idris AM, Mansoor S, Bedford ID, Dhawan P, Rishi N, Siwatch SS, Abdel-Salam AM, Brown JK, Zafar Y, Markham PG: Diversity of DNAß: a satellite molecule associated with some monopartite begomoviruses. Virology 2003, 312:106-121.

2. Saunders K, Bedford ID, Briddon RW, Markham PG, Wong SM, Stanley J: A unique virus complex caused Ageratum yellow vein disease. Proc Natl Acad Sci USA 2000, 97:6890-6895.

3. Zhou X, Xie Y, Tao X, Zhang Z, Li Z, Fauquet CM: Characterization of DNA $\beta$ associated with begomoviruses in China and evidence for co-evolution with their cognate viral DNA-A. J Gen Virol 2003, 84:237-247. 
4. Briddon RW, Mansoor S, Bedford ID, Pinner MS, Saunders K, Stanley J: Identification of DNA components required for induction of cotton leaf curl disease. Virology 2001, 285:234-243.

5. Cui $X$, Tao $X$, Xie $Y$, Fauquet $C M$, Zhou X: A DNA $\beta$ associated with Tomato yellow leaf curl China virus is required for symptom induction. J Virol 2004, 78:13966-13974.

6. Saunders K, Norman A, Gucciardo S, Stanley J: The DNA $\beta$ satellite component associated with Ageratum yellow vein disease encodes an essential pathogenicity protein ( $\beta C 1)$. Virology 2004, 324:37-47.

7. Yang JY, Iwasaki M, Machida C, Machida Y, Zhou X, Chua $\mathrm{NH}$ : $\beta C 1$, the pathogenicity factor of TYLCCNV, interacts with AS1 to alter leaf development and suppress selective jasmonic acid responses. Genes Dev 2008, 22:2564-2577.

8. Dry I, Krake L, Mullineaux P, Rezaian A: Regulation of tomato leaf curl viral gene expression in host tissues. Mol Plant Microbe Interact 2000, 13:529-537.

9. Eagle PA, Hanley-Bowdoin L: Cis elements that contribute to geminivirus transcriptional regulation and the efficiency of DNA replication. J Virol 1997, 71:6947-6955.

10. Eini O, Behjatnia SA, Dogra S, Dry IB, Randles JW, Rezaian MA: Identification of sequence elements regulating promoter activity and replication of a monopartite begomovirus-associated DNA $\beta$ satellite. J Gen Virol 2009, 90:253-260.

11. Shung CY, Sunter J, Sirasanagandla SS, Sunter G: Distinct viral sequence elements are necessary for expression of Tomato golden mosaic virus complementary sense transcripts that direct $A L 2$ and $A L 3$ gene expression. Mol Plant Microbe Interact 2006, 19:1394-1405.

12. Sunter $G$, Bisaro DM: Identification of a minimal sequence required for activation of the Tomato golden mosaic virus coat protein promoter in protoplasts. Virology 2003, 305:452-462.

13. Sunter G, Bisaro DM: Regulation of a geminivirus coat protein promoter by AL2 protein (TrAP): evidence for activation and depression mechanisms. Virology 1997, 232:269-280.

14. Guan C, Zhou X: Phloem specific promoter from a satellite associated with a DNA virus. Virus Res 2006, 115:150-157.

15. Guo W, Jiang T, Zhang X, Li G, Zhou X: Molecular variation of satellite DNA $\beta$ molecules associated with Malvastrum yellow vein virus and their role in pathogenicity. App/ Environ Microbiol 2008, 74:1909-1913.

16. Bian C, Ruan Q, Peng Z, Ji H, Jiang L, Li J, Yuan L: Cloning, expression and characterization of the putative nuclear transport factor 2 (NTF2) gene from moss Conocephalum conicum (L.) Dum. Mol Biol Rep 2011, 38:2023-2032.

17. Choudhury SR, Roy S, Sengupta DN: A comparative study of cultivar differences in sucrose phosphate synthase gene expression and sucrose formation during banana fruit ripening. Postharvest Biol Tec 2009, 54:15-24.

18. Du H, Zhang Z, Li J: Isolation and functional characterization of a waterlogging-induced promoter from maize. Plant Cell Rep 2010, 29:1269-1275

19. Ramos PL, Fuentes AD, Quintana Q, Castrillo G, Guevara-Gonzalez RG, Pera $R$, Rivera-Bustamante RF, Pujol M: Identification of the minimal sequence required for vascular-specific activity of Tomato mottle Taino virus replication-associated protein promoter in transgenic plants. Virus Res 2004, 102:125-132.

20. Armentero MT, Horwitz M, Mermod N: Targeting of DNA polymerase to the adenovirus origin of DNA replication by interaction with nuclear factor I. Proc Natl Acad Sci USA 1994, 91:11537-11541.

21. DePamphilis ML: How transcription factors regulate origins of DNA replication in eukaryotic cells. Trends Cell Biol 1993, 3:161-167.

22. Ito K, Asano M, Hughes $P$, Kohzaki H, Kerppola T, Curran T, Murakami Y, Ito Y: c-Jun stimulates origin-dependent DNA unwinding by polyomavirus large T antigen. EMBO J 1996, 15:5636-5646.

23. Lue NF, Kornberg RD: A possible role for the yeast TATA-element-binding protein in DNA replication. Proc Natl Acad Sci USA 1993, 90:8018-8022.

24. Hur J, Choi E, Buckley KJ, Lee S, Davis KR: Identification of a promoter motif involved in Curtovirus sense-gene expression in transgenic Arabidopsis. Mol Cells 2008, 26:131-139.

25. Sardo L, Lucioli A, Tavazza M, Masenga V, Tavazza R, Accotto PG, Noris E: An RGG sequence in the replication-associated protein (Rep) of Tomato yellow leaf curl Sardinia virus is involved in transcriptional repression and severely impacts resistance in Rep-expressing plants. J Gen Virol 2011, 92:204-209.
26. Tu J, Sunter G: A conserved binding site within the Tomato golden mosaic virus AL-1629 promoter is necessary for expression of viral genes important for pathogenesis. Virology 2007, 367:117-125.

27. Ding C, Qing L, Li Z, Qian Y, Zhou X: Genetic determinants of symptoms on viral DNA satellites. Appl Environ Microbiol 2009, 75:5380-5389.

28. Dinant S, Ripoll C, Pieper M, David C: Phloem specific expression driven by wheat dwarf geminivirus $\mathrm{V}$-sense promoter in transgenic dicotyledonous species. Physiol Plantarum 2004, 121:108-116.

29. Xie Y, Liu Y, Meng M, Chen L, Zhu Z: Isolation and identification of a super strong plant promoter from Cotton leaf curl Multan virus. Plant Mol Biol 2003, 53:1-14

30. Yin $Y, C$ hen $L$, Beachy R: Promoter elements required for phloemspecific gene expression from the RTBV promoter in rice. Plant J 1997, 12:1179-1188

31. Yang Y, Li R, Qi M: In vivo analysis of plant promoters and transcription factors by agroinfiltration of tobacco leaves. Plant J 2000, 22:543-551.

32. Dry IB, Krake LR, Rigden JE, Rezaian MA: A novel subviral agent associated with a geminivirus: the first report of a DNA satellite. Proc Natl Acad Sci USA 1997, 94:7088-7093.

33. Pratap D, Kashikar AR, Mukherjee SK: Molecular characterization and infectivity of a Tomato leaf curl new Delhi virus variant associated with newly emerging yellow mosaic disease of egg plant in India. Virol J 2011, 8:305

34. Horsch RB, Fry JE, Hoffman NL, Eichholtz D, Rogers SG, Fraley RT: A simple and general method for transferring genes into plants. Science 1985 227:1229-1231.

35. Jefferson RA, Kavanagh TA, Bevan MW: GUS fusions: $\beta$-glucuronidase as a sensitive and versatile gene fusion marker in higher plants. EMBO J 1987 , 6:3901-3907.

36. Glauert AM: Practical Methods in Electron Microscopy. Specimen Preparation Mater Sci 1972, I:1.

doi:10.1186/1743-422X-9-234

Cite this article as: Zhang et al:: Characterization of sequence elements from Malvastrum yellow vein betasatellite regulating promoter activity and DNA replication. Virology Journal 2012 9:234.

\section{Submit your next manuscript to BioMed Central and take full advantage of:}

- Convenient online submission

- Thorough peer review

- No space constraints or color figure charges

- Immediate publication on acceptance

- Inclusion in PubMed, CAS, Scopus and Google Scholar

- Research which is freely available for redistribution
C) Biomed Central 